\title{
ADDITIVE FUNCTIONALS AND EXCURSIONS OF KUZNETSOV PROCESSES
}

\author{
HACÈNE BOUTABIA
}

Received 16 September 2004 and in revised form 28 June 2005

Let $B$ be a continuous additive functional for a standard process $\left(X_{t}\right)_{t \in \mathbb{R}_{+}}$and let $\left(Y_{t}\right)_{t \in \mathbb{R}}$ be a stationary Kuznetsov process with the same semigroup of transition. In this paper, we give the excursion laws of $\left(X_{t}\right)_{t \in \mathbb{R}_{+}}$conditioned on the strict past and future without duality hypothesis. We study excursions of a general regenerative system and of a regenerative system consisting of the closure of the set of times the regular points of $B$ are visited. In both cases, those conditioned excursion laws depend only on two points $X_{g^{-}}$ and $X_{d}$, where $] g, d\left[\right.$ is an excursion interval of the regenerative set $M$. We use the $\left(F_{D_{t}}\right)$ predictable exit system to bring together the isolated points of $M$ and its perfect part and replace the classical optional exit system. This has been a subject in literature before (e.g., Kaspi (1988)) under the classical duality hypothesis. We define an "additive functional" for $\left(Y_{t}\right)_{t \in \mathbb{R}}$ with $B$, we generalize the laws cited before to $\left(Y_{t}\right)_{t \in \mathbb{R}}$, and we express laws of pairs of excursions.

\section{Introduction}

Let $X$ be a standard process, and let $M$ be a closed random and homogeneous subset of $\mathbb{R}_{+}$. Kaspi [8] constructs an additive functional $B$ associated to $M$ and gives, under the classical duality hypothesis, the probability measures allowing the law of excursions to be associated to $B$ with respect to the $\sigma$-algebra $K=\sigma\left(Z_{t}: t \in \mathbb{R}_{+}\right)$, known to start at $x$ and end at $y\left(Z_{t}=X_{S_{t}}\right.$ where $\left.S_{t}=\inf \left\{u: B_{u}>t\right\}\right)$. The purpose of this paper is to give, without duality, the conditional law $P^{x, y}$ of the excursion straddling an arbitrary random time, given the initial state $x$ and the final state $y$, as regular probabilities in terms of the $\left(F_{D_{t}}\right)$-predictable exit measures for $M$ and also for a regenerative system consisting of the closure of the set of times the regular points of an arbitrary continuous additive functional are visited. We also give the conditional laws of pairs of excursions for a Markov process with random birth and death $\left(Y_{t}\right)_{t \in \mathbb{R}}$ having the same semigroup as $X$. In this respect, we define an "additive functional" for $\left(Y_{t}\right)_{t \in \mathbb{R}}$ and we extend this result concerning the probability measures $P^{x, y}$ to $\left(Y_{t}\right)_{t \in \mathbb{R}}$.

In Section 2, we introduce our notations, preliminaries, and Maisonneuve's result [12] on the strict past conditioning with respect to the filtration $\left(F_{D_{t}}\right)$. In Section 3, we 
construct the probability measures $P^{x, y}$, which allows the law of the excursion to straddle an arbitrary random time, given the initial state $x$ and the final state $y$. Section 4 deals with excursions associated to a continuous additive functional $B$. The measures $P^{x, y}$ which govern these excursion are the same as defined in Section 3 corresponding to the regenerative set $M$ where contiguous intervals are of the form $] S_{t^{-}}, S_{t}[, t$ is a time of discontinuity of $S$. Laws of excursions and of pairs of excursions for $\left(Y_{t}\right)_{t \in \mathbb{R}}$ are discussed in Section 5.

\section{Notations and preliminaries}

Let $\left(\Omega, F, F_{t}, X_{t}, \theta_{t}, P^{x}\right)$ be a canonical realization for a Borel standard semigroup $\left(P_{t}\right)$ with lifetime $\zeta$, and let $M$ be a closed random and homogeneous subset of ]0, $\zeta$ [ such that $R=\inf M$ is $F^{*}$-measurable, where $F^{*}$ is the universal completion of the $\sigma$-algebra $F^{0}=$ $\sigma\left(X_{t}: t \in \mathbb{R}_{+}\right)$. We assume that the state space $E$ is Lusinian, and we denote by $\mathscr{E}$ its $\sigma$ algebra of Borel sets. The cimetry point $\delta$ is outside of $E$. We denote by $G^{0}$ the set of the left endpoints of the contiguous intervals of $M$.

Let $\left({ }^{\circ} P^{x}\right)_{x \in E \cup \delta}$ be the family of $\left(F_{t}^{D}\right)$-predictable exit measures for the process $\left(X_{t}^{D}\right)=$ $\left(X_{D_{t}}\right)$ in the sense of Maisonneuve [11], and let $\mu$ be a fixed law on $E$. Then $\left({ }^{\circ} P^{x}\right)_{x \in E \cup \delta}$ is a universally measurable family of $\sigma$-finite measures on $\left(\Omega, F^{*}\right)$, under which the process $\left(X_{t}\right)$ is Markov with respect to $\left(P_{t}\right)$.

For all $t \in \mathbb{R}_{+}$, let $k_{t}$ be the killing operator at $t$ defined by $k_{t}(\omega)(s)=\omega(s)$ if $s<t$ and $\delta$ if $s \geq t$. If $t \in \mathbb{R}_{+}, \omega, \omega^{\prime} \in \Omega$, we denote by $\omega / t / \omega^{\prime}$ the trajectory $\bar{\omega} \in \Omega$ such that $\bar{\omega}(s)=\omega(s)$ (or $\left.k_{s}(\omega)\right)$ if $s<t$ and $\omega^{\prime}(s-t)$ if $s \geq t$.

Let $T$ be a random time on $(\Omega, F)$ such that $T<D_{T}$ on $\{T<\zeta\}\left(D_{t}=\inf \{s \geq t: s \in\right.$ $M\}$ for $t \in \mathbb{R}_{+}$with the convention $\left.\inf \varnothing=+\infty\right)$, and let $g=\sup \{s \leq T: s \in M\}$ and $d=\inf \{s>T: s \in M\}$. Then with the following notations:

$$
\begin{gathered}
A^{\omega}\left(\omega^{\prime}\right)=T(\omega)-g\left(\omega / g(\omega) / \omega^{\prime}\right), \quad C^{\omega}=\left\{0 \leq A^{\omega}<R\right\}, \\
v(B \mid A)=\frac{v(B \cap A)}{v(A)} \quad\left(\frac{0}{0}=\frac{\infty}{\infty}=0\right)
\end{gathered}
$$

if $v$ is a measure on $\left(\Omega, F^{*}\right)$, we have the basic Maisonneuve formula [12].

For almost all $\omega \in\{g<\infty\}$ we have (if $\delta$ is nonabsorbent)

$$
P\left(f\left(\theta_{g}\right) \mid F_{g^{-}}^{D}\right)(\omega)={ }^{\circ} P^{X_{g^{-}}^{D}(\omega)}\left(f \mid C^{\omega}\right)
$$

for every $F^{*}$-measurable function $f \geq 0$, where $P$ is the probability measure defined by $P(f)=\int P^{x}(f) \mu(d x)$. If we assume that $\delta$ is absorbent, then this formula is true on $\{g<$ $\zeta\}$ instead of $\{g<\infty\}$.

Note that if $T$ is an $\left(F_{t}^{D}\right)=\left(F_{D_{t}}\right)$-stopping time, we can replace $C^{\omega}$ by the condition $A^{\omega}<R$, and if $T \in G^{0}$ on $\left\{X_{T} \in E\right\}$, the set $C^{\omega}$ can be replaced by the condition $A^{\omega}=0$.

\section{The excursion straddling $T$}

For the conditional law of the excursion $e=k_{R} \circ \theta_{g}$ straddling $T$, with respect to $F_{g^{-}}^{D}$ and $\theta_{d}$, we assume that $\delta$ is absorbent. In this respect, we consider, for $(x, y) \in E \times E$, 
the measures $H^{x}, H_{1}^{x}$ and $P^{x, y}$ on $\left(\Omega, F^{*}\right)$ "defined by"

$$
\begin{gathered}
H^{x}={ }^{\circ} P^{x}\left(k_{R} \in \cdot \mid X_{R}=x\right), \quad H_{1}^{x}={ }^{\circ} P^{x}\left(\cdot ; X_{R} \neq x\right), \\
P^{x, y}=H_{1}^{x}\left(k_{R} \in \cdot \mid X_{R}=y\right) \quad \text { if } x \neq y .
\end{gathered}
$$

Since $\left(\Omega, F^{0}\right)$ is a U-space, and according to a classical lemma of Doob, the measures $P^{x, y}$ can be chosen measurable for the pair $(x, y)$.

Proposition 3.1. Let $x \in E, A \in F^{*}$ such that $0<{ }^{\circ} P^{x}(A)<+\infty$, and let the probability measure $\mu^{x}$ be defined on $\left(\Omega, F^{*}\right)$ by $\mu^{x}={ }^{\circ} P^{x}(\cdot \mid A)$. Then for almost all $\omega \in \Omega$,

$$
\mu^{x}\left(f \circ k_{R} \mid \theta_{R}\right)(\omega)=M^{x, X_{R(\omega)}}\left(f \mid\left(\cdot / \zeta / \theta_{R}(\omega)\right) \in A\right)
$$

where

$$
M^{x, y}(f)=P^{x, y}(f) I_{\{x \neq y\}}+H^{x}(f) I_{\{x=y\}} .
$$

Proof. Note that formula (3.2) means

$$
\begin{aligned}
\mu^{x}\left(f \circ k_{R} \mid \theta_{R}\right)(\omega) & =P^{x, X_{R(\omega)}}\left(f \mid B^{\omega}\right) \quad \text { for } a \cdot a \omega \in\left\{X_{R} \neq x\right\}, \\
\mu^{x}\left(f \circ k_{R} \mid \theta_{R}\right)(\omega) & =H^{x}\left(f \mid B^{\omega}\right) \quad \text { for } a \cdot a \omega \in\left\{X_{R}=x\right\}, \\
\text { where } B^{\omega} & =\left\{\left(\cdot / \zeta / \theta_{R}(\omega)\right) \in A\right\},
\end{aligned}
$$

which follows from the Markov property at time $R$ with an argument of monotone classes, the definitions of $P^{x, y}$ and $H^{x}$, and the fact that $\omega=\left(k_{R}(\omega) / \zeta \circ k_{R}(\omega) / \theta_{R}(\omega)\right)$ for all $\omega \in \Omega$.

The following theorem gives the conditional law of the excursion $e$ with respect to $F_{g^{-}}^{D}$ and $\theta_{d}$.

Theorem 3.2. For all $\omega \in\{g<\infty\}$, let the subset of $\Omega$ be defined by $U_{d}^{\omega}=\left\{\omega^{\prime} \in \Omega\right.$ : $\left.\left(\omega^{\prime} / \zeta\left(\omega^{\prime}\right) / \theta_{d}(\omega)\right) \in C^{\omega}\right\}$. Then

(1) for almost all $\omega \in\left\{X_{d} \neq X_{g^{-}}^{D} ; g<\zeta ; d-g<\infty\right\}$,

$$
P\left(f(e) \mid F_{g^{-}}^{D}, \theta_{d}\right)(\omega)=P^{X_{g^{-}}^{D}(\omega), X_{d}(\omega)}\left(f \mid U_{d}^{\omega}\right)
$$

(2) for almost all $\omega \in\left\{X_{d}=X_{g^{-}}^{D}, g<\zeta\right\}$,

$$
P\left(f(e) \mid F_{g^{-}}^{D}, \theta_{d}\right)(\omega)=H^{X_{g^{-}}^{D}(\omega)}\left(f \mid U_{d}^{\omega}\right)
$$

It follows that if $T$ is an $\left(F_{D_{t}}\right)$-stopping time such that $T \in G^{0}$ on $\left\{X_{T} \in E\right\}$, then formulas (3.5) and (3.6) hold without conditioning by $U_{d}^{\omega}$ in the right sides. 


\section{Additive functionals and excursions of Kuznetsov processes}

Proof. Let $Z$ be a positive $F_{g^{-}}^{D}$-measurable random variable carried by $\{g<\zeta ; d-g<\infty\}$, and let $\varphi$ be a positive $F^{0}$-measurable function. We have to prove that

$$
P\left(f(e) Z \varphi\left(\theta_{d}\right) I_{\left\{X_{d} \neq X_{g^{-}}^{D}\right\}}\right)=\int_{\left\{X_{d} \neq X_{g^{-}}^{D}\right\}} P(d \omega) Z(\omega) \varphi\left(\theta_{d}\right)(\omega) P^{X_{g^{-}}^{D}(\omega), X_{d}(\omega)}\left(f \mid U_{d}^{\omega}\right) .
$$

By formula (2.2) and the definition of $\mu^{x}$ with $x=X_{g^{-}}^{D}(\omega)$ and $A=C^{\omega}$, the left side of formula (3.7) is equal to

$$
\int P(d \omega) Z(\omega) \mu^{x}\left(f \circ k_{R} \varphi\left(\theta_{R}\right) I_{\left\{X_{R} \neq x\right\}}\right)
$$

which by formula (3.2) is equal to

$$
\int P(d \omega) Z(\omega) \int_{\left\{X_{R} \neq x\right\}} \mu^{x}\left(d \omega^{\prime}\right) \varphi\left(\theta_{R}\right)\left(\omega^{\prime}\right) P^{x, X_{R\left(\omega^{\prime}\right)}}\left(f \mid\left(\cdot / \zeta / \theta_{R}\left(\omega^{\prime}\right)\right) \in A\right),
$$

and using formula (2.2) again, we obtain the right side of (3.7). Formula (3.6) is argued in the same manner using formula (3.4).

Remark 3.3. Maisonneuve [12] gives several examples where the set $C^{\omega}$ is independent of $\omega$. In these cases Theorem 3.2 implies that the excursion $e$ is conditionally independent of $F_{g^{-}}^{D}$ and $\theta_{d}$ given $X_{g^{-}}^{D}$ (resp., $X_{g^{-}}^{D}$ and $X_{d}$ ) on $\left\{X_{g^{-}}^{D}=X_{d} ; g<\zeta\right\}$ (resp., $\left\{X_{g^{-}}^{D} \neq X_{d} ; g<\right.$ $\zeta ; d-g<\infty\})$.

Remark 3.4. Theorem 3.2 contains results of Kaspi [8, Section 5] under duality hypothesis. In fact if $M$ is perfect, then $X_{g^{-}}^{D}=X_{g^{-}}$and $F_{g^{-}}^{D}=F_{g^{-}}$. If $T$ is the beginning of the set $\left\{t \in \mathbb{R}_{+}:\left(X_{t^{-}}, X_{t}\right) \in J\right\}$, where $J \in \mathscr{E} \otimes \mathscr{E}$, then with the assumption that ${ }^{\circ} P^{x}\left(X_{0^{-}} \neq\right.$ $x)=0$, the conditions $0<g(\omega)<T(\omega)$ and $\theta_{g}(\omega) \in C^{\omega}$ are equivalent to the condition $\theta_{g}(\omega) \in\left\{\left(X_{0^{-}}, X_{0}\right) \notin J ; 0<T<R\right\}$, and formula (2.2) becomes

$$
P\left(f\left(\theta_{g}\right) \mid F_{g^{-}}\right)={ }^{\circ} P^{X_{g^{-}}}\left(f \mid\left(X_{0^{-}}, X_{0}\right) \notin J ; 0<T<R\right) \quad \text { on }\{0<g<T<\zeta\} .
$$

According to the same argument used in Theorem 3.2 and the fact that $T=T \circ k_{R}$ on $\{T<R\}$ and $R=\zeta \circ k_{R}$, formula (3.5) becomes

$$
P\left(f(e) \mid F_{g^{-}}, \theta_{d}\right)=P^{X_{g^{-}}, X_{d}}\left(f \mid\left(X_{0^{-}}, X_{0}\right) \notin J ; 0<T<\zeta\right) \quad \text { on }\left\{X_{g^{-}} \neq X_{d} ; 0<g<T<\zeta\right\}
$$

and formula (3.6) becomes

$$
\begin{aligned}
& P\left(f(e) \mid F_{g^{-}}, \theta_{d}\right) \\
& \quad=H^{X_{g^{-}}}\left(f \mid\left(X_{0^{-}}, X_{0}\right) \notin J ; 0<T<\zeta\right) \text { on }\left\{X_{g^{-}}=X_{d} ; 0<g<T<\zeta ; d-g<\infty\right\} .
\end{aligned}
$$

\section{Excursions associated to an additive functional}

Let $\left(B_{t}\right)$ be a continuous additive functional and let $C=\left\{x: P^{x}(R=0)=1\right\}$ be its fine support, where $R$ is the perfect exact terminal time $\inf \left\{u: B_{u}>0\right\}$. We associate to the 
right inverse $S_{t}=\left\{u: B_{u}>t\right\}$ of $\left(B_{t}\right)$ the notations $Z_{t}=X_{S_{t}}, M_{t}=F_{S_{t}}$, and $\bar{\theta}_{t}=\theta_{S_{t}}$. It is well known that the process $Z=\left(\Omega, F, M_{t}, Z_{t}, \bar{\theta}_{t}, P^{x}\right)$ is strong Markov with semigroup $\left(\overline{P_{t}}\right) \triangleq\left(P_{S_{t}}\right)$ and takes values on $\left(C, C \cap \mathscr{E}^{*}\right)$ (cf. Jacobs [7]).

In this section, we assume that $\delta$ is nonabsorbent and we consider the random homogeneous set $M=\left\{t+R \circ \theta_{t}: t \in \mathbb{R}_{+}\right\}$and its family of $\left(F_{D_{t}}\right)$-predictable exit measures $\left({ }^{0} P^{x}\right)_{x \in E \cup\{\delta\}}$. If $S_{t^{-}} \neq S_{t}$, then $D_{S_{t^{-}}}=S_{t}$. The excursion associated to $t$ is then defined by

$$
e_{t}=k_{R} \circ \theta_{S_{t^{-}}}= \begin{cases}X_{S_{t^{-}}+s} & \text { if } s<S_{t}-S_{t^{-}} \\ \delta & \text { if } s \geq S_{t}-S_{t^{-}} .\end{cases}
$$

We denote by $\left(K_{t}\right)_{t \in \mathbb{R}_{+}}$the filtration, where $K_{t}$ is the intersection of the $P^{\pi}$ completions of the $\sigma$-algebra $K_{t^{+}}^{0}$ where $\pi$ is in the set of all the bounded measures on $E ;\left(K_{t}^{0}\right)$ is the natural filtration of the process $\left(Z_{t}\right)$.

For the following lemma we put $K_{0^{-}}=F_{0}$ by convention.

Lemma 4.1. Let $T$ be a $\left(K_{t}\right)$-stopping time such that $S_{T^{-}}<S_{T}$ a.s. Then

$$
F_{\left(S_{T^{-}}\right)^{-}}=K_{T^{-}} .
$$

Proof. According to the fact that $S_{T^{-}}$is not an isolate point of $M$, we have $F_{\left(S_{T^{-}}\right)^{-}}=$ $F_{\left(S_{T^{-}}\right)^{-}}^{D}$. Since $K_{t}^{0}=\sigma\left(X_{S_{u^{-}}}^{D}: u \leq t\right) \subset F_{\left(S_{t^{-}}\right)}^{D}$, then every $\left(K_{t}\right)$-predictable process is $F_{\left(S_{t^{-}}\right)^{-}}^{D}$ predictable, which implies that $K_{T^{-}} \subset F_{\left(S_{T^{-}}\right)^{-}}$. For the inclusion $F_{\left(S_{T^{-}}\right)^{-}} \subset K_{T^{-}}$, since $F_{0} \subset$ $K_{T^{-}}$it suffices to prove that $A=B \cap\left\{t<S_{T^{-}}\right\} \in K_{T^{-}}$for all $B \in F_{t}$.

Note that $S_{T^{-}}=\sup _{r<T}\left\{S_{r}: r \in \mathbb{Q}_{+}\right\}$, which implies that

$$
\left\{t<S_{T^{-}}\right\}=\bigcup_{r \in \mathbb{Q}_{+}}\{r<T\} \cap\left\{t<S_{r}\right\}, \quad A=\bigcup_{r \in \mathbb{Q}_{+}}\left(B \cap\left\{t<S_{r}\right\} \cap\{r<T\}\right),
$$

where $\mathbb{Q}_{+}$is the set of positive rationals. For all $u \leq t$ we have $X_{u}=Z_{B_{u}}$, then $F_{t} \subset K_{B_{t}}$, which implies that

$$
B \cap\left\{t<S_{r}\right\}=B \cap\left\{B_{t} \leq r\right\} \in K_{r}
$$

and $A \in K_{T^{-}}$. The proof is complete.

The following theorem which gives the conditional law of the excursion $e_{T}$ associated to a $\left(K_{t}\right)$-stopping time $T$, with respect to the $\sigma$-algebra $K$ generated by $K_{t}(t \geq 0)$, was proved by Kaspi [8] under the duality hypothesis.

Theorem 4.2. Let $T$ be a finite $\left(K_{t}\right)$-stopping time. Then

(1) $S_{T^{-}}$is an $\left(F_{D_{t}}\right)$-stopping time,

(2) it is assumed that $S_{T^{-}} \neq S_{T}$ and $Z_{T^{-}} \neq Z_{T}$ a.s., then the following formula:

$$
P\left(f\left(e_{T}\right) \mid K\right)=P^{Z_{T^{-}}, Z_{T}}(f)
$$

holds for every positive and $F^{*}$-measurable function $f$.

Proof. (1) If $t \in \mathbb{R}_{+}$, then $\left\{u<S_{t^{-}}\right\}=\left\{D_{u} \leq S_{t}\right\}=\left\{B_{D_{u}} \leq t\right\} \in F_{u}^{D}$ and $S_{t^{-}}$is an $\left(F_{D_{u}}\right)$ stopping time. 
Let $\left(T_{n}\right)_{n \in \mathbb{N}}$ be the nondecreasing dyadic approximation of $T$, then

$$
\left\{S_{T_{n}} \leq u\right\}=\bigcup_{k \in \mathbb{N}}\left\{S_{\left(k / 2^{n}\right)} \leq u\right\} \cap\left\{\frac{k}{2^{n}} \leq T<\frac{k+1}{2^{n}}\right\} .
$$

Since $\left\{k / 2^{n} \leq T<(k+1) / 2^{n}\right\} \in K_{\left((k+1) / 2^{n}\right)^{-}} \subset F_{S_{\left((k+1) / 2^{n}\right)^{-}}^{D}}^{D} \subset F_{S_{\left((k+1) / 2^{n}\right)}^{D}}^{D}$, then

$$
\left\{S_{\left(k / 2^{n}\right)} \leq u\right\} \cap\left\{\frac{k}{2^{n}} \leq T<\frac{k+1}{2^{n}}\right\} \in F_{u}^{D}, \quad\left\{S_{T_{n}} \leq u\right\} \in F_{u}^{D},
$$

which implies that $\left\{S_{T^{-}} \leq u\right\} \in F_{u}^{D}$.

(2) For every continuous $\left(K_{t}\right)$-adapted process $U \geq 0$, and for every positive $F^{*}$ measurable function $\varphi$, we have by formula (3.5) with $S_{T^{-}}$instead of $T$ and the fact that $K_{T^{-}}=F_{\left(S_{T^{-}}\right)^{-}}^{D}$ the following:

$$
P\left(f\left(e_{T}\right) U_{T} \varphi\left(\bar{\theta}_{T}\right)\right)=P\left(P^{Z_{T^{-}}, Z_{T}}(f) U_{T} \varphi\left(\bar{\theta}_{T}\right)\right) .
$$

Formula (4.5) follows from the fact that $K$ is generated by $K_{T^{-}}$and $\bar{\theta}_{T}$.

\section{Excursions of Kuznetsov processes}

Let $W$ be the set of applications $w: \mathbb{R} \mapsto E \cup\{\delta\}$ which satisfies the following properties: there exists an open interval of $\mathbb{R}$ on which $w$ is $E$-valued right-continuous with left limits and out of which $w$ equals $\delta$. We denote by $\left(Y_{t}\right)_{t \in \mathbb{R}}$ the coordinate process on $W$. Let $\left(\varphi_{t}^{0}\right)_{t \in \mathbb{R}}$ be the natural filtration of $\left(Y_{t}\right)_{t \in \mathbb{R}}$ and let $\mathscr{G}^{0}=\mathscr{V}_{t \in \mathbb{R}} \varphi_{t}^{0}$. Then the birth and the death times of $\left(Y_{t}\right)_{t \in \mathbb{R}}$ are, respectively,

$$
\begin{aligned}
& \alpha=\inf \left\{t \in \mathbb{R}: Y_{t} \in E\right\} \quad(\inf \varnothing=+\infty), \\
& \beta=\sup \left\{t \in \mathbb{R}: Y_{t} \in E\right\} \quad(\sup \varnothing=-\infty) .
\end{aligned}
$$

We define the families of operators on $W$ by

$$
\begin{array}{cl}
\tau_{t}: W \longmapsto \Omega \text { such that } \tau_{t} w(s)=w(s+t) & \text { for } s \in \mathbb{R}_{+}, t \in \mathbb{R}, \\
\sigma_{t}: W \longmapsto W \text { such that } \sigma_{t} w(s)=w(s+t) & \text { for } s, t \in \mathbb{R} .
\end{array}
$$

Note that $X_{s} \circ \tau_{t}=Y_{t+s}$ on $\left\{Y_{t} \in E\right\}$ and $\sigma_{t} \circ \sigma_{u}=\sigma_{t+u}$ for $t, u \in \mathbb{R}, s \in \mathbb{R}_{+}$. Let $\eta$ be an excessive measure with respect to $\left(P_{t}\right)$ and let $Q$ be the Kuznetsov measure on $W$ that corresponds to $\left(\eta,\left(P_{t}\right)\right)$ (cf. $\left.[9,10]\right)$. We denote by $\mathscr{G}_{t}$ and $\mathscr{G}$ the $Q$-completions of $\varphi_{t}^{0}$ and $\varphi^{0}$, and we assume that the semigroup $\left(P_{t}\right)$ satisfies "les hypothèses droites de Meyer." It follows by [13] that the process $Y=\left(W, \mathscr{G}_{,} \mathscr{G}_{t},\left(Y_{t}\right)_{t \in \mathbb{R}}, \tau_{t}, \alpha, \beta, Q\right)$ is stationary (i.e., $\sigma_{t}(Q)=Q$ ) and strong Markov with semigroup $\left(P_{t}\right)$.

For the generalization of Theorem 4.2, we consider the additive functionals $B$ and $S$ given in the previous section. We also denote by $B$ the random measure on $W$, carried by ]$\alpha, \beta[$ such that

$$
\left.\left.B_{s} \circ \tau_{t}=B\right] t, t+s\right] \quad \text { on }\left\{Y_{t} \in E\right\}, \forall s>0, t \in \mathbb{R}
$$


We assume that the characteristic measure $v_{B} \triangleq Q \int_{0}^{1} I_{\left\{Y_{t} \in \cdot\right\}} B(d t)$ of $B$ is purely excessive for the semigroup $\left(\overline{P_{t}}\right)$ (i.e., $\int \overline{P_{t}} f(x) v_{B}(d x) \rightarrow 0$ as $t \rightarrow \infty$ if $\left.v_{B}(f)<\infty\right)$. It was shown in [9] that $Q$ a.e. $B] \alpha, t]<\infty$ for all $t>\alpha$.

Let $\left(V_{t}\right)_{t \in \mathbb{R}}$ be the nondecreasing process defined on $W$ by

$$
\left.\left.V_{t}=\alpha+B\right] \alpha, t\right] \quad \text { on }\{\alpha<t\}, \quad V_{t}=\alpha \quad \text { on }\{t \leq \alpha\}
$$

and let $\left(U_{t}\right)_{t \in \mathbb{R}}$ be the right-continuous inverse of $\left(V_{t}\right)_{t \in \mathbb{R}}$, that is,

$$
U_{t}=\inf \left\{u>\alpha: V_{u}>t\right\}
$$

We also denote by $M$ the closed random subset of ] $\alpha, \beta$ [ defined by $M=\bigcup_{\alpha<t<\beta}\{t+R$ 。 $\left.\tau_{t}\right\}$ which verifies the following property of homogeneity (cf. [4]):

$$
(M-t) \cap] 0, \infty\left[=M \circ \tau_{t} \quad \text { on }\left\{Y_{t} \in E\right\} .\right.
$$

Remark 5.1. (1) If $\alpha=-\infty,\left\{u>\alpha: V_{u}>t\right\}=\varnothing$, and $U_{t}=+\infty$, then $\alpha>-\infty$ on $\{\alpha<$ $\left.U_{t}<\beta\right\}$.

(2) $U_{t}=\alpha$ on $\{t \leq \alpha\}$.

For $t \in \mathbb{R}$, let $\Phi_{t}=Y_{U_{t}}, \overline{\mathscr{G}}_{t}=\mathscr{G}_{U_{t}}, \bar{\tau}_{t}=\tau_{U_{t}}, \mathscr{H}_{t}^{0}=\sigma\left(\Phi_{u}: u \leq t\right)$, and $\mathscr{H}^{0}=\mathscr{V}_{t \in \mathbb{R}} \mathscr{H}_{t}^{0}$. We denote by $\mathscr{H}_{t}$ (resp., $\mathscr{H}$ ) the $Q$-completion of $\mathscr{H}_{t^{+}}^{0}$ (resp., $\left.\mathscr{H}^{0}\right)$. Note that for all the following formulas, the $\sigma$-finiteness of $Q$ is guaranteed by the argument used in [1]. It is not hard to show that $\left(\Phi_{t}\right)$ has the same properties as $\left(Z_{t}\right)$ and that the following result holds.

Proposition 5.2. (1) The process $\left(U_{t}\right)$ is right-continuous, has left limits, and satisfies $U_{t}=$ $U_{\beta}$ for all $t \geq \beta$ Q a.e.

(2) $\left(U_{t}\right)$ is $\left(\bar{G}_{t}\right)$-adapted.

(3) For all $t \in \mathbb{R}$ and $s>0$,

(a) $U_{t}=\alpha+S_{t-\alpha} \circ \tau_{\alpha}$ on $\{-\infty<\alpha<t\}$,

(b) $V_{t+s}=V_{t}+B_{s} \circ \tau_{t}$ on $\left\{Y_{t} \in E\right\}$ and $U_{t+s}=U_{t}+S_{s} \circ \bar{\tau}_{t}$ on $\left\{\alpha<U_{t}<\beta\right\}$.

(4) On $\left\{U_{t} \neq U_{t^{-}}\right\}$, ] $U_{t^{-}}, U_{t}$ [ is a contiguous interval of $M$.

If $U_{t} \neq U_{t^{-}}$, let $E_{t}$ be the excursion associated to $B$ and defined by

$$
E_{t}(w)(s)= \begin{cases}Y_{U_{t^{-}}+s}(w) & \text { if } 0 \leq s<U_{t}(w)-U_{t^{-}}(w) \\ \delta & \text { if } s \geq U_{t}(w)-U_{t^{-}}(w) .\end{cases}
$$

According to the previous proposition, the process $\left(V_{t}\right)_{t \in \mathbb{R}}$ has got the same role as $B$ for the process $\left(Y_{t}\right)_{t \in \mathbb{R}}$. We say that $\left(V_{t}\right)_{t \in \mathbb{R}}$ is an "additive functional" for $\left(Y_{t}\right)_{t \in \mathbb{R}}$. We have the extension of Theorem 4.2 on $W$.

Theorem 5.3. (1) The process $\Phi=\left(W, \Phi_{t}, \mathscr{G}_{,}, \bar{G}_{t}, \bar{\tau}_{t}, Q\right)$ is strong Markov in the sense that for all $\left(\bar{G}_{t}\right)$-stopping time $T$ and $s>0$,

$$
Q\left(f\left(\Phi_{T+s}\right) \mid \bar{G}_{T}\right)=\bar{P}_{s}\left(f, \Phi_{\mathrm{T}}\right) \quad \text { on }\left\{\alpha<U_{T}<\beta\right\}
$$

for every positive and F-measurable function $f$. 
(2) Assume that $T_{1}$ is a finite $\left(\mathcal{H}_{t}\right)$-stopping time such that $U_{T_{1}} \neq U_{T_{1}^{-}}$and $\Phi_{T_{1}} \neq \Phi_{T_{1}^{-}} Q$ a.e. Then

$$
Q\left(F\left(E_{T_{1}}\right) \mid \mathscr{H}\right)=P^{\Phi_{T_{1}^{-}}, \Phi_{T_{1}}}(F) \quad \text { on }\left\{\alpha<U_{T_{1}}<\beta\right\}
$$

for all $F^{*}$-measurable $F \geq 0$.

Proof. If $T \equiv t$ is constant, formula (5.8) follows from the Markov property of the process $\left(Y_{t}\right)_{t \in \mathbb{R}}$ at time $U_{t}$ and the fact that $\Phi_{t+s}=Z_{s} \circ \bar{\tau}_{t}$ and $\bar{\tau}_{t+s}=\bar{\theta}_{s} \circ \bar{\tau}_{t}$ on $\left\{\alpha<U_{t}<\beta\right\}$. This formula is also true for $T_{n}$ instead of $T$, where $\left(T_{n}\right)$ is the decreasing dyadic approximation of $T$, which extends for a general $T$ by the right continuity of the processes $\left(\Phi_{t}\right)$, $\left(U_{t}\right)$, and $\left(\bar{\tau}_{t}\right)$.

Formula (5.9) is argued in the same manner as (4.5) by using [1, formula (30)].

We consider now the time-reversed process $\left(\hat{Y}_{t}\right)_{t \in \mathbb{R}}=\left(Y_{(-t)^{-}}\right)_{t \in \mathbb{R}}$. It is an $E$-valued right-continuous process with left limits on $] \hat{\alpha}, \hat{\beta}[=]-\beta,-\alpha[$, and which is equal to $\delta$ outside of $] \hat{\alpha}, \hat{\beta}$. As in $[1,13]$, we assume that $\left(\hat{Y}_{t}\right)$ is also Markov with respect to another standard semigroup $\left(\hat{P}_{t}\right)$ satisfying "les hypothèses droites de Meyer," which implies the strong Markov property and the existence of exit systems. The measure

$$
\hat{\eta}(B)=Q\left(\hat{Y}_{t} \in B ; \hat{\alpha}<t<\hat{\beta}\right)
$$

is $\left(\widehat{P}_{t}\right)$-excessive and the stationarity of $\left(\hat{Y}_{t}\right)$ is guaranteed. Let $\hat{\tau}_{t}, \hat{\mathscr{G}}_{t}, \hat{B}, \hat{S}, \hat{V}_{t}, \hat{U}_{t}$, and $\hat{E}_{t}$ be the analog of $\tau_{t}, \mathscr{G}_{t}, B, S, V_{t}, U_{t}$, and $E_{t}$ corresponding to $\left(\hat{Y}_{t}\right)$. As previously we assume that $Q$ a.e. $\hat{B}] \hat{\alpha}, t]<\infty$ for all $t>\hat{\alpha}$. For the process $\left(\Psi_{t}\right)=\left(\hat{Y}_{\hat{U}_{t}}\right)$ and the random subset

$$
\widehat{M}=\bigcup_{\hat{\alpha}<t<\hat{\beta}}\left\{t+R \circ \hat{\tau}_{t}\right\}
$$

of $] \hat{\alpha}, \hat{\beta}\left[\right.$, we have the analog of Theorem 5.3. In particular if we design by $\hat{\mathscr{H}}_{\text {and }} \hat{\mathscr{H}}_{t}$ the Q-completions of $\sigma\left(\Psi_{u}: u \in \mathbb{R}\right)$ and $\sigma\left(\Psi_{u}: u \leq t\right)_{+}$, respectively, and by $\hat{P}^{x, y}$ the measure defined as $P^{x, y}$ in terms of the exit measures ${ }^{0} \widehat{P}^{x}$ of $\widehat{M}$ for the canonical realization of $\left(\hat{P}_{t}\right)$, we have the following formula:

$$
Q\left(F\left(\hat{E}_{T_{2}}\right) \mid \hat{\mathscr{H}}\right)=\hat{P}^{\Psi_{T_{2}^{-}}, \Psi_{T_{2}}}(F) \quad \text { on }\left\{\hat{\alpha}<\hat{U}_{T_{2}}<\hat{\beta}\right\}
$$

for all finite $\left(\hat{\mathscr{H}}_{t}\right)$-stopping time $T_{2}$ such that $\hat{U}_{T_{2}} \neq \hat{U}_{T_{2}^{-}}$and $\Psi_{T_{2}^{-}} \neq \Psi_{T_{2}^{-}} Q$ a.e., and for every positive $F^{*}$-measurable function $F$.

For the following theorem which gives the conditional law of pairs of excursions, we consider the family of probability measures

$$
Q^{x, y, z, u}=P^{x, y} \otimes \hat{P}^{z, u} .
$$

Theorem 5.4. Let $T_{1}$ (resp., $T_{2}$ ) be as in (5.9) (resp., (5.12)). Assume that the following hypotheses are satisfied:

(1) $\sigma\left(U_{T_{1}^{-}}\right) \cap \Lambda \subset \hat{\mathscr{H}}_{T_{2}^{-}}$,

(2) $\sigma\left(\hat{U}_{T_{2^{-}}}\right) \cap \Lambda \subset \mathscr{H}_{T_{1}^{-}}$, 
where $\Lambda=\left\{\alpha<-\hat{U}_{T_{2}^{-}} \leq U_{T_{1}^{-}}<\beta\right\}$. Then the following formula:

$$
Q\left(H\left(E_{T_{1}}, \widehat{E}_{T_{2}}\right) \mid \mathscr{H} \cap \hat{\mathscr{H}}\right)=Q^{\Phi_{T_{1^{-}}}, \Phi_{T_{1}}, \Psi_{T_{2}^{-}}, \Psi_{T_{2}}}(H) \text { on } \Lambda
$$

holds for every positive and $F^{*} \otimes F^{*}$-measurable function $H$.

Since $\mathscr{G}_{\left(U_{T_{1}^{-}}\right)^{-}}^{D}=\mathscr{H}_{T_{1}^{-}}$and $\hat{\mathscr{G}}_{\left(\hat{U}_{T_{2}^{-}}\right)^{-}}=\hat{\mathscr{H}}_{T_{2}^{-}}$, the hypothesis (1) (resp., (2)) means that the trace on $\Lambda$ of the $\sigma$-algebra generated by $U_{T_{1}^{-}}$(resp., $\hat{U}_{T_{2}^{-}}$) is contained in the strict past of $\hat{U}_{T_{2}^{-}}$(resp., $U_{T_{1}^{-}}$) with respect to the filtration $\left(\varphi_{t}^{D}\right)$ (resp., $\left(\hat{G}_{t}^{\hat{D}}\right)$ ).

Proof. We have to prove that

$$
Q\left(F\left(E_{T_{1}}\right) \hat{F}\left(\widehat{E}_{T_{2}}\right) Z I_{\Lambda}\right)=Q\left(P^{\Phi_{T_{1}-}, \Phi_{T_{1}}}(F) \hat{P}^{\Psi_{T_{2}^{-}}, \Psi_{T_{2}}}(\hat{F}) Z I_{\Lambda}\right)
$$

for all positive and $F^{*}$-measurable functions $F, \hat{F}$, and for every positive $\mathscr{H} \cap \hat{\mathscr{H}}$ measurable random variable $Z$. Since $\hat{\tau}_{\hat{U}_{T_{2}^{-}}}=\theta_{U_{T_{1}^{-}}+\hat{U}_{T_{2}^{-}}} \circ \hat{\tau}_{-U_{T_{1}^{-}}}$on $\Lambda$, and since $\hat{\tau}_{-U_{T_{1}^{-}}}$is $\mathscr{G}_{\left(U_{T_{1}^{-}}\right)^{-}}$-measurable and $\mathscr{G}_{\left(U_{T_{1}^{-}}\right)^{-}}^{D}=\mathscr{H}_{T_{1}^{-}}$, then $\sigma\left(\hat{\tau}_{\hat{U}_{T_{2}^{-}}}\right) \cap \Lambda \subset \mathscr{H}_{T_{1}^{-}}\left(\mathscr{G}_{t}^{D}=\mathscr{G}_{D_{t}} \supset \mathscr{G}_{t}\right.$, where $D_{t}=\inf \{s>t: s \in M\}$ on $W$, for $\left.t \in \mathbb{R}\right)$. By using the same argument, we prove that $\sigma\left(\Psi_{T_{2}^{-}}, \Psi_{T_{2}}\right) \cap \Lambda \subset \mathscr{H}_{T_{1}^{-}}, \sigma\left(U_{T_{1}^{-}}\right) \cap \Lambda \subset \hat{\mathscr{H}}_{T_{2}^{-}}$, and $\sigma\left(\Phi_{T_{1}^{-}}, \Phi_{T_{1}}\right) \cap \Lambda \subset \hat{\mathscr{H}}_{T_{2}^{-}}$.

The Markov property at time $T_{1}$ and formula (5.9) implied that for every positive $\mathcal{H}_{T_{1}^{-}}$measurable random variable $Z_{1}$, and for every positive and $F^{0}$-measurable function $\varphi$,

$$
Q\left(F\left(E_{T_{1}}\right) \hat{F}\left(\hat{E}_{T_{2}}\right) Z_{1} \varphi\left(\bar{\tau}_{T_{1}}\right) I_{\Lambda}\right)=Q\left(P^{\Phi_{T_{1-}}, \Phi_{T_{1}}}(F) \hat{F}\left(\hat{E}_{T_{2}}\right) Z_{1} \varphi\left(\bar{\tau}_{T_{1}}\right) I_{\Lambda}\right)
$$

and according to the fact that $\mathscr{H}$ is generated by $\mathscr{H}_{T_{1}^{-}}$and $\bar{\tau}_{T_{1}}$, we have

$$
Q\left(F\left(E_{T_{1}}\right) \hat{F}\left(\widehat{E}_{T_{2}}\right) Z I_{\Lambda}\right)=Q\left(P^{\Phi_{T_{1-}}, \Phi_{T_{1}}}(F) \hat{F}\left(\hat{E}_{T_{2}}\right) Z I_{\Lambda}\right) .
$$

Formula (5.15) follows by using formulas (5.12) and (5.17).

\section{References}

[1] H. Boutabia, Lois conditionnelles des excursions d'un processus de Markov à naissance et mort aléatoires [Conditional excursion laws for a Markov process with random birth and death times], Portugal. Math. 56 (1999), no. 2, 239-255 (French).

[2] H. Boutabia and B. Maisonneuve, Lois conditionnelles des excursions markoviennes [Conditional distributions of Markov excursions], Séminaire de Probabilités, XXVI, Lecture Notes in Math., vol. 1526, Springer, Berlin, 1992, pp. 162-166.

[3] C. Dellacherie, B. Maisonneuve, and P. A. Meyer, Probabilités et potentiel, chapter 17-19, Hermann, Paris, 1992.

[4] R. K. Getoor, Killing a Markov process under a stationary measure involves creation, Ann. Probab. 16 (1988), no. 2, 564-585.

[5] R. K. Getoor and M. J. Sharpe, Two results on dual excursions, Seminar on Stochastic Processes, 1981 (Evanston, Ill., 1981), Progr. Prob. Statist., vol. 1, Birkhäuser, Massachusetts, 1981, pp. 31-52.

[6] Excursions of dual processes, Adv. in Math. 45 (1983), no. 3, 259-309. 
[7] P. A. Jacobs, Excursions of a Markov process induced by continuous additive functionals, Z. Wahrsch. Verw. Gebiete 44 (1978), no. 4, 325-336.

[8] H. Kaspi, Excursion laws of Markov processes in classical duality, Ann. Probab. 13 (1985), no. 2, 492-518.

[9] _ Random time changes for processes with random birth and death, Ann. Probab. 16 (1988), no. 2, 586-599.

[10] S. E. Kuznetsov, Construction of Markov processes with random times of birth and death, Theory Probab. Appl. 18 (1973), no. 3, 571-575.

[11] B. Maisonneuve, Systèmes de sortie $\left(\mathscr{F}_{D_{t}}\right)$ prévisibles [( $\left.\mathscr{F}_{D_{t}}\right)$-predictable exit systems], Probab. Theory Related Fields 80 (1989), no. 3, 395-405 (French).

[12] Excursions chevauchant un temps aléatoire quelconque [Excursions spanning a random time], Astérisque 236 (1996), 215-226 (French).

[13] J. B. Mitro, Exit systems for dual Markov processes, Z. Wahrsch. Verw. Gebiete 66 (1984), no. 2, 259-267.

Hacène Boutabia: Département de Mathématiques, Faculté des Sciences, Université Badji Mokhtar, BP 12, Annaba 23000, Algeria

E-mail address: hboutabia@hotmail.com 


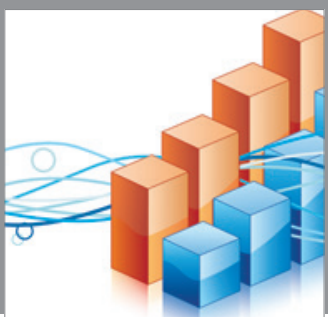

Advances in

Operations Research

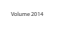

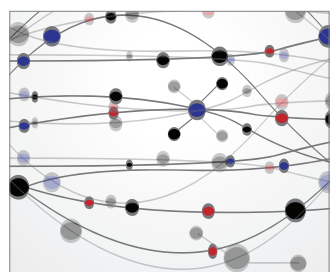

\section{The Scientific} World Journal
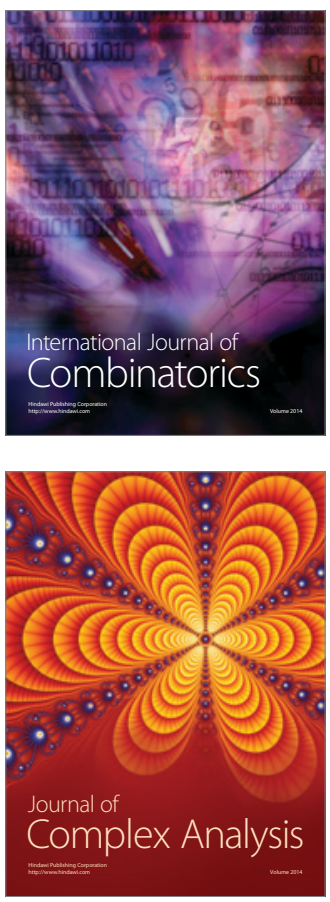

International Journal of

Mathematics and

Mathematical

Sciences
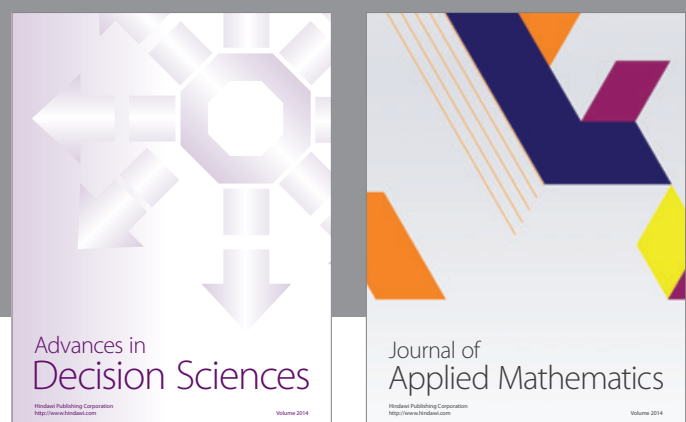

Journal of

Applied Mathematics
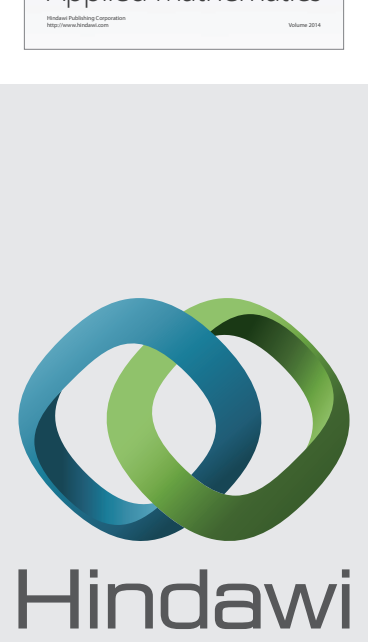

Submit your manuscripts at http://www.hindawi.com
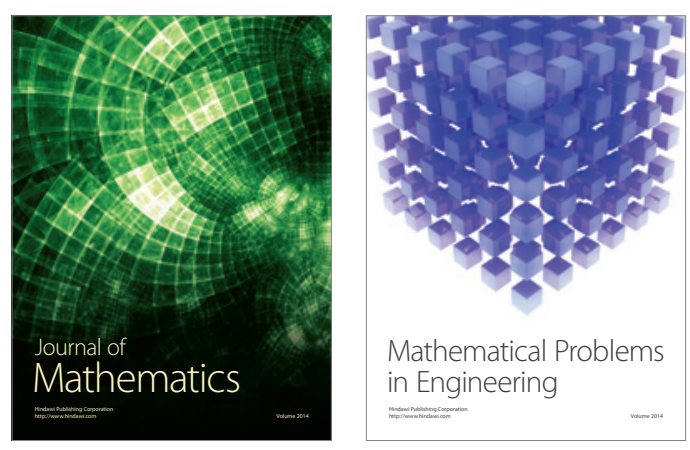

Mathematical Problems in Engineering
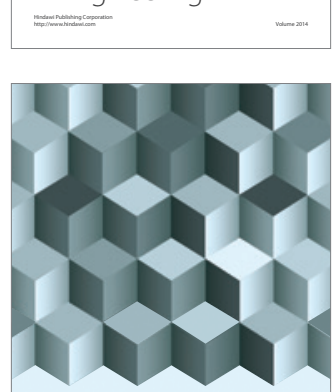

Journal of

Function Spaces
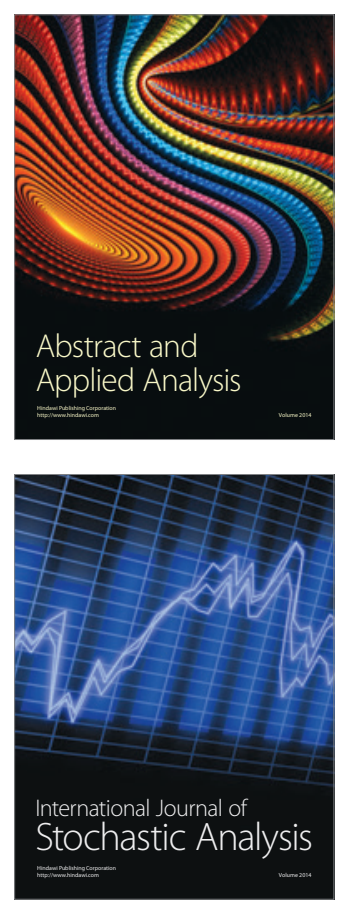

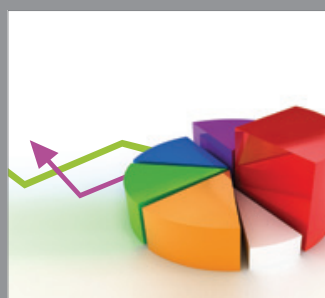

ournal of

Probability and Statistics

Promensencen
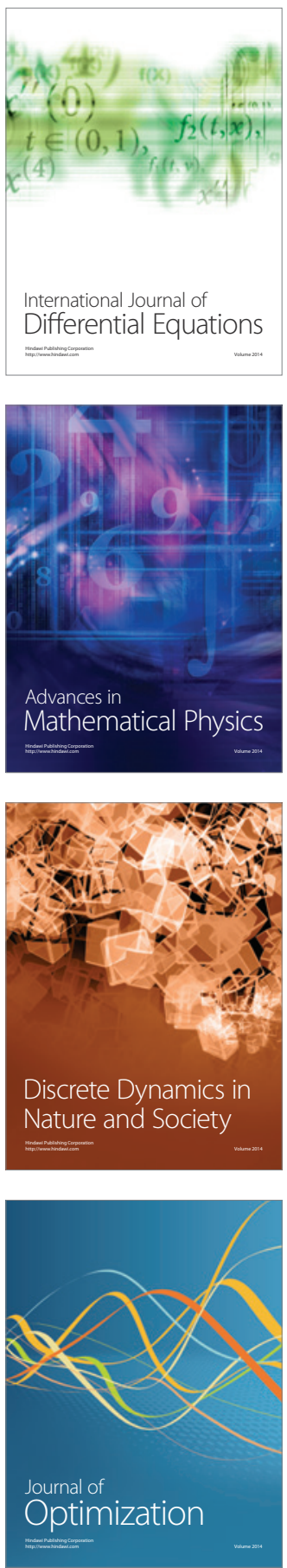